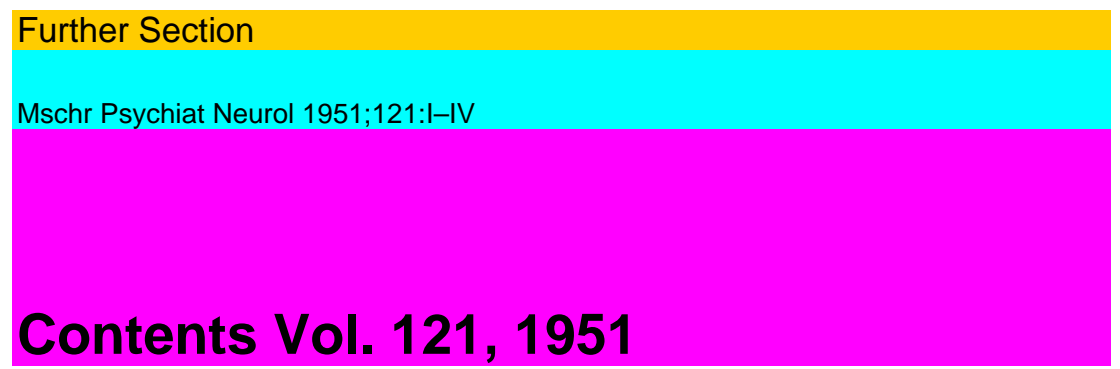

Monatsschrift für

\title{
Psychiatrie und Neurologie
}

Revue Mensuelle de Psychiatrie et de

Neurologie Monthly Review of Psychiatry and

Neurology

\author{
M. BLEULER, Zürich \\ L.VAN BOGAERT, Anvers \\ E. CARP, Leiden \\ 0. DIETHELM, New York \\ R. GJESSING, Oslo \\ E. GRÜNTHAL, Bern \\ H. HELWEG, Kopenhagen \\ COLLABORATORES:
}

B. JACOBOWSKY, Upsala E. KAHN, New Haven

E. KRETSCHMER, TübingenG. LANGFELDT, Oslo

J. LEPINE, Lyon A. LEWIS, London J. LHERMITTE, Paris

F. MOREL, Geneve

G. PAMBOUKIS, Athen

KURT SCHNEIDER, Heidelbe

H. SCHOU, Dianalund

J. STAEHELIN, Basel

H. STECK, Lausanne

G. STOERRING, Düsseldorf

C. I. URECHIA, Sibiu 
EDITOR:

J. KLAESI, Bern

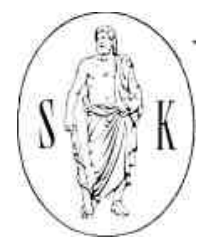

Vol. 121

1951

BASEL (Schweiz)

S. KARGER

NEW

YORK

Alle Rechte vorbehalten Tous droits reserves 
Printed in Switzerland Druck von Gasser und Cie. AG. in Basel Gliche's: Aberegg-Steinor \& Cie. AG. Bern

\section{INDEX}

Altwegs, F., Electromyographisehe Untersuchungen über die Wirkungsweise des Parpanits

Ajuriaguerra, J. de, vide Boga'ert, L. v.

Bischoff, A., Über eine therapeutische Verwendung der sogenannten „WeckAmine" in der Behandlung schizophrener Erregungszustände $\quad$.... 329

Bogaerl, L. v., M. David, J. de Ajuriaguerra, H. Hecaen et Ä, Talairach, XX.D'im Syndrome degeneratif du groupe de la dystonie ä debut cerebelleuxcorrespondant ä une abiotropliie spinostriee avec degenerescence duSysteme olfactif.

143

Bogaert, L. v., XXI. Sur un Syndrome extra-pyramidal caracterise par uneatonie extreme et des reflexes de decerebration au cours d'un ictereprolonge grave du nouveau-ne, ä debut tardif et en dehors d'une iso-immunisation decelable - Analyse anatomoclinique .................................. 265

Christoffel, H., Physiognomik und Physiologie ....................................................................... 133

David, M., vide Bogaert, L. v.

Descamps, L., vide FranQois, J.

Eichhorn, 0. und JV. Moschik de Reya, Zur Pathogenese, Klinik und Begutachtung psychisch bedingter Unfallsfolgen

Feremutsch, K., Die Variabilität der cytoarchitektonischen Struktur des menschlichen Hypothalamus

Francois, J. et L, Descamps, Heredo-ataxie par degenerescence spino-ponto-cerebelleuse avec manifestations tapeto-retiniennes et clochleo-vesti-bulaires. (Avec planches I et II) ..........

Gäl, P., Zur Psychotherapie der Schizophrenie

Gomirato, G. et F. R. Gratlarola, Meningo-encephalite allergique

Graltarola, F. R., vide Gomirato, G.

Haberland, IC, Ein Fall von Panarteritis disseminata necrotica (periarteritisnodosa). Klinische und pathologisch-anatomische Beschreibung mitbesonderer Berücksichtigung der Veränderungen im Nervensystem . .

Halpern, L., Impairment of Orientation to Pictures in Cerebral Diseases and in Chronic Schizophrenias.

Hecaen, H., vide Bogaert, L. v.

Heim, H., Arteritis des Hirns bei maligner Nephrosklerose und chronischer

diffuser Glomerulonephritis

Heimann, H., Erfahrungen mit der frontalen Lobotomie bei Schizophrenen

Juba, A., Das Ependymom der Cauda-aequina-Gegend

Kahn, JE., Randbemerkungen zum schizophrenen Erleben

Lehoczky, T. und A-Sai-Haläsz, Doppelseitiges frontopolares bösartiges Ependymom

Maas, 0., Observations on „Myotonia acquisita".....

Martin, F. et B. Rilliet, Gliome centro-bulbaire et centro-medullaire cervicalayant simule le tableau clinitrue d'une sclerose en placjue. Etüde ana-tomo-chnique.

Mittenecker, E., Eine neue quantitative Methode in der Spraehanalyse und

ihre Anwendung bei Schizophrenen

Moschik de Reya, N., vide Eichhorn, 0.

Remy, M., Zur Frage der photogenen Epilepsie 
Rilliet, B., vide Martin, F.

Rozanski, J., Apparition d'une evolution suraigue mortelle de la sclerose en

plaques apres une seule injection de vaccin antityphoiiditpie.

Sai-Haläsz, A., vide Lehoczky, T.

Scheele, H., Organische Merkunfähigkeit oder Hysterie

Simma, K., Über Thalamusveränderungen bei progressiver Pai'alyse ....

297

Stern, A., Über Emprosthotonus

Talairach, R., vide Bogaert, L. v.

Wormser, P., Ophthalmoplegia interna und andere Augensymptome bei Poliomyelitis Heine-Medin.

\section{Libri 58}

199

262

326

383

Varia 264

388 